

Investigación original

\title{
Sensibilidad y especificidad de la amilasemia como ayuda diagnóstica inicial para Pancreatitis Aguda en pacientes que acudieron por dolor abdominal agudo al Servicio de Emergencia del Hospital Vozandes - Quito entre los meses de julio del año 2006 y junio del año 2007.
}

\section{Viteri Cevallos, Diego.}

Médico Rural. Hospital Cantonal de Sangolquí, Ecuador.

\section{Resumen}

Objetivo: Validar la medición de amilasa sérica como herramienta diagnóstica inicial en pacientes que acudieron al Servicio de Emergencia por presentar dolor abdominal agudo.

Lugar: Servicio de Emergencia del Hospital Vozandes - Quito

Fecha: Pacientes que acudieron a Emergencia con dolor abdominal agudo entre julio del año 2006 y junio del año 2007

Pacientes y Métodos: Se realizó un estudio Retrospectivo de Validación de Herramientas Diagnósticas. Se incluyeron entre 15 y 64 años de edad. La muestra que se utilizó para este estudio fueron 955 pacientes. Para el análisis estadístico de la amilasa sérica se calculó la sensibilidad, especificidad, valor predictivo, razón por verosimilitud y el índice kappa de Cohen. Además se obtuvo la curva ROC y el AUC de la misma. Se utilizó como patrón de oro para el análisis el diagnóstico de pancreatitis aguda.

Resultados: En el intervalo de amilasa sérica de 176 a 200 mg/dl (mejor punto de corte) se obtuvo una sensibilidad de 96\% (IC 95\%: 86-99), especificidad de $97 \%$ (IC 95\%: 96-98), valor predictivo positivo de $68 \%$ (IC 95\% 56-78), valor predictivo negativo de 99\% (IC 95\%: 99-100), LR+ de 37, LR- de 25 y kappa de 0.78.

Revista electrónica publicada por el Departamento de Farmacología de la Escuela de

Medicina de la Universidad de Costa Rica, 2060 San José, Costa Rica. ® All rights

reserved. 
Conclusiones: En pacientes que acuden a Emergencia con dolor abdominal agudo y sospecha de posible pancreatitis aguda es válida la medición de amilasemia como prueba inicial de ayuda diagnóstica.

Palabras Clave: amilasa, pancreatitis, validación diagnóstica

Recibido: Febrero 2009. Aprobado: Marzo 2009. Publicado: Marzo 2009.

\begin{abstract}
Objective: To determine the validity of the serum amylase measurement as initial diagnostic tool in patients who went to the Emergency Service with acute abdominal pain.

Place: Emergency Service of Vozandes Hospital - Quito

Date: From July 2006 to June 2007

Patients and Methods: A restrospective study of diagnostic tools validation was carried out. It included patients between 15 and 64 years of age.

The sample used for this study was 955 patients. Sensitivity, specificity, predictive value, likehood ratio and Cohen's kappa index were calculated for the statistical analysis. It was used as gold standard for the analysis of acute pancreatitis diagnosis.

Results: In the interval of 176 to $200 \mathrm{mg} / \mathrm{dl}$ of serum amylase (best cut value), it was obtained: sensibility of 96\% (IC 95\%: 86 - 99), specificity of 97\% (IC 95\%: $96-98$ ), positive predictive value of $68 \%$ (IC 95\%: $56-78$ ), negative predictive value of $99 \%$ (IC 95\%: $99-100)$, LR+ of 37, LR- of 25, and kappa of 0.78 .

Conclusions: In patients who go to the Emergency Service with acute abdominal pain and the suspicion of possible acute pancreatitis, it is valid the use of amilasemia measurement as initial test of diagnostic aid.
\end{abstract}

Keywords: amylase, pancreatitis, diagnostic validation

\section{INTRODUCCIÓN}

El uso inadecuado de pruebas diagnósticas y su aplicación sin un sustento científico ha aumentado en los últimos años. En la práctica médica diaria existe un sinnúmero de procedimientos y exámenes de laboratorio que son realizados en forma innecesaria sin el conocimiento de su aplicabilidad y utilidad real, lo que en muchas ocasiones impide lograr los objetivos planteados previamente con cada paciente que acude a la consulta médica.

El dolor abdominal agudo (DAA) definido como un síndrome caracterizado por una instauración en pocas horas, brusco y progresivo, el cual abarca muchos aspectos clínicos, de diagnóstico y manejo, plantea un desafío médico urgente y se presenta como un síntoma guía en varias patologías y requiere una correcta evaluación en el Servicio de Emergencia [3] .

Revista electrónica publicada por el Departamento de Farmacología de la Escuela de 
En USA fue causa de 7 millones de visitas al hospital en el año 2005 [4]; alrededor del $5 \%$ de adultos con DAA que acuden al Servicio de Emergencia requieren ingreso al hospital y un porcentaje menor necesita tratamiento quirúrgico[5].

Dentro del diagnóstico diferencial del paciente con DAA, en una investigación realizada en 384 pacientes, se encontró como principales patologías las siguientes: dolor abdominal inespecífico (14\%), gastritis (10\%), cólico biliar $(10 \%)$, pancreatitis aguda (7\%), patología urológica (8\%), apendicitis aguda (7\%) y otras [7].

En el manejo inicial del DAA la historia clínica y un examen físico adecuados permiten una evaluación diagnóstica correcta de cada paciente, el uso de laboratorio y métodos de imagen complementan la información obtenida para confirmar la posible patología que se sospecha [8].

Es importante indicar que en pacientes con dolor en epigastrio la amilasa es útil como prueba esencial dentro del diagnóstico de inicio en el Servicio de Emergencia [9].

En numerosas ocasiones es poco útil recurrir a estudios complementarios, ya que el diagnóstico clínico es suficiente, aunque en muchos casos el dolor abdominal es inespecífico, por lo que algunos expertos recomiendan que es mejor esperar, observar el desarrollo del cuadro y si es indispensable volver a realizar una anamnesis y examen físico exhaustivo; dependiendo de la gravedad y posible etiología en pocos pacientes es necesario efectuar dentro de las primeras 24 horas estudios especiales 0 tratamiento quirúrgico [10].

Dentro del diagnóstico diferencial del DAA se encuentra la pancreatitis aguda (PA), cuya sospecha inicial es clínica, pero es necesario complementar su evaluación con el uso de pruebas de laboratorio, dentro de las cuales está la amilasa sérica [11], una enzima producida por:

- Glándulas salivales (60\%).

- Páncreas (40\%).

- Hígado y trompas de Falopio (menos del $1 \%)$.

Presenta dos isómeros: la P-isoamilasa cuya producción es exócrina en el páncreas y la S-isoamilasa cuya producción es a nivel salival, tiene una vida media de 2 horas, su metabolismo se produce en el sistema retículo endotelial $(75 \%)$ y su excreción depende de su nivel en sangre y el aclaramiento renal $(25 \%)$ [12].

Cuando existen procesos de inflamación del páncreas, el nivel plasmático de ésta enzima se eleva dentro de 2 a 12 horas, pero disminuye paulatinamente conforme se presentan los síntomas del paciente [13]; otros estudios refieren que presenta un pico de elevación dentro de 12 a 72 horas y sus niveles disminuyen aproximadamente en una semana. No tiene buena especificidad ni sensibilidad y serviría de poca ayuda en establecer la gravedad de la enfermedad [14].

En varias investigaciones se señala que la amilasa sérica con cifras de $300 \mathrm{mg} / \mathrm{dl}$ o más tiene una sensibilidad de $85-89 \%$ y especificidad de $91-95 \%$ para pancreatitis en pacientes con DAA [16].

Revista electrónica publicada por el Departamento de Farmacología de la Escuela de 
Otros autores refieren una sensibilidad de 75 a $92 \%$ y una especificidad de 20 a $60 \%[14]$; en otro estudio se observó una especificidad de 80 a 85\%[17], también se indica que la amilasa sérica para ayuda diagnóstica en la sospecha de pancreatitis aguda tiene que subir sus niveles por encima de $330 \mathrm{U} / \mathrm{L}[18]$, entre 3 a 4 veces lo normal o hasta 10 veces su valor [1].

En un estudio realizado por Muñoz y cols., se indica que la lipasa sérica tiene mayor sensibilidad (86-100\%) y especificidad (50-99\%) en comparación con la amilasa sérica, es útil pedir esta prueba, ya que se eleva 18 horas luego del inicio del cuadro, tiene un pico a las 24 horas y se mantiene detectable por 8 a 14 días [14].

Existen ocasiones en que la amilasa sérica a pesar de una inflamación pancreática se encuentra normal o disminuida como en: pancreatitis necrótica extensa, alcoholismo, hepatitis, enfermedad hepática severa, insuficiencia pancreática 0 fibrosis quística [11]. En un estudio realizado se indica que puede haber amilasa normal en el $19 \%$ de pacientes con pancreatitis aguda [21].

Puede existir elevación de amilasa sérica en: obstrucción del intestino delgado, rotura de aneurisma aórtico, embarazo ectópico roto, perforación de víscera hueca, peritonitis generalizada, cetoacidosis diabética, espasmo del esfínter de Oddi por opiáceos, isquemia mesentérica [22], gastrectomía parcial, obstrucción de conductos pancreáticos, trauma cerebral, colecistitis aguda, acidosis láctica, anorexia nerviosa, bulimia, insuficiencia renal (hasta 5 veces lo normal) [17], perforación esofágica, cáncer de pulmón, mama y estómago, mieloma, consumo de tabaco[12], pero esta elevación no supera tres veces el rango establecido [36].

El número de pruebas especiales innecesarias realizadas en pacientes con dolor abdominal está en aumento, su uso debe sustentarse en guías de medicina basadas en evidencia, es importante tener una secuencia apoyándose en una sospecha clínica y diagnóstica, para lograr de esta manera disminuir la cantidad de exámenes de laboratorio realizados y utilizar en forma correcta los recursos de salud dentro de cada centro de salud o unidad hospitalaria [2].

\section{OBJETIVO}

Validar la medición de amilasa sérica como herramienta diagnóstica inicial en pacientes que acudieron al servicio de Emergencia por dolor abdominal $y$ sospecha inicial de pancreatitis aguda.

\section{MATERIALES Y METODOS}

Se realizó un estudio Retrospectivo de Validación de Herramientas Diagnósticas.

Se incluyó a los pacientes entre 15 y 64 años que acudieron al servicio de Urgencias del Hospital Vozandes con dolor abdominal agudo, en los cuales se realizó la prueba de amilasa sérica; excluyéndose a aquellos que acudieron por trauma abdominal 0 que presentaron antecedentes de pancreatitis crónica. 
La muestra inicial de pacientes que se utilizó para esta investigación fueron 1012 pacientes, la cual abarcó todo el universo, por lo que no se realizó cálculo del tamaño muestral.

Durante los meses de enero a mayo del año 2008 se seleccionó de los libros de pacientes del Servicio de Emergencia del Hospital Vozandes - Quito, las historias clínicas de los pacientes que acudieron por dolor abdominal a dicho servicio.

Se seleccionó las historias clínicas de los pacientes que cumplieron los criterios de inclusión y mediante una hoja con un formato preestablecido de recolección de datos, se obtuvo en la Unidad de Estadística de la institución la información individual (edad, género, diagnóstico inicial y definitivo, amilasa sérica) correspondiente a cada historia.

Los descriptivos de las variables categóricas (género, diagnóstico inicial y definitivo) están expresados mediante frecuencias absolutas y porcentajes, y los de las variables cuantitativas (edad y valor de amilasa sérica) a través de medidas de tendencia central: media, mediana y moda, y de dispersión: desviación estándar, varianza, rango, kurtosis y sesgo[24,25].

Para el análisis estadístico de la amilasa sérica se estableció 10 puntos de corte y se obtuvo intervalos de confianza al 95\% para los siguientes índices de probabilidad:

- Sensibilidad (S)

- Especificidad (E)

- Valor predictivo positivo (VP+)

- Valor predictivo negativo (VP-)
- Indice de máxima verosimilitud (LR+o LR-)

- Indice kappa de Cohen

Posteriormente, en base de los diferentes puntos de corte con que se encontró la sensibilidad y especificidad, se elaboró una curva ROC y se obtuvo el área bajo la curva o AUC de la misma.

Se utilizó como patrón de oro para la investigación los criterios clínicos y de laboratorio de la Sociedad Americana de Gastroenterología para el diagnóstico de Pancreatitis Aguda [34]:

1. Clínicos: dolor abdominal, náusea y vómito.

2. Laboratorio: elevación de amilasa (normal $75-100 \mathrm{mg} / \mathrm{dl}$ ) y lipasa (normal 80-110 mg/dl) mayor de 3 veces su valor establecido.

Los pacientes que presentaron pancreatitis como sospecha inicial fueron hospitalizados y se complementó el diagnóstico definitivo con ecografía de abdomen y tomografía computarizada, junto a exámenes de laboratorio para establecer la severidad y pronóstico en cada caso (ej: criterios de Ramson).

Los resultados obtenidos por las variables se muestran con cuadros de distribución de frecuencia y gráficos.

Para la sistematización, procesamiento y análisis de datos se utilizó los programas: Microsoft Excel (Microsoft Office para Windows versión 2003) y SPSS (Windows español versión 11.5).

Revista electrónica publicada por el Departamento de Farmacología de la Escuela de 


\section{RESULTADOS}

Durante el período de tiempo en que se realizó la investigación 1012 pacientes acudieron al Servicio de Emergencia por dolor abdominal agudo y fueron realizados amilasa sérica.

De los 955 pacientes que cumplieron los criterios de inclusión e ingresaron al estudio, más del $50 \%$ fueron mujeres; los pacientes entre 25 y 44 años abarcaron el grupo de edad con mayor porcentaje.



Fuente: Datos del autor

La variable amilasa sérica presentó un valor de la media de $138 \mathrm{mg} / \mathrm{dl}$ que varió considerablemente en relación a la mediana (75 mg/dl), el sesgo y la kurtosis se presentaron elevados, por lo que se realizó un histograma para identificar mejor la distribución de los pacientes.

El $60 \%$ de los pacientes presentaron durante su valoración inicial dolor abdominal en estudio y enfermedad ácido péptica, en menor porcentaje se observó casos de enfermedad diarreica aguda, gastroenteritis, colelitiaisis, colecistitis y pancreatitis aguda (Ver tabla 1 y gráfico 2).

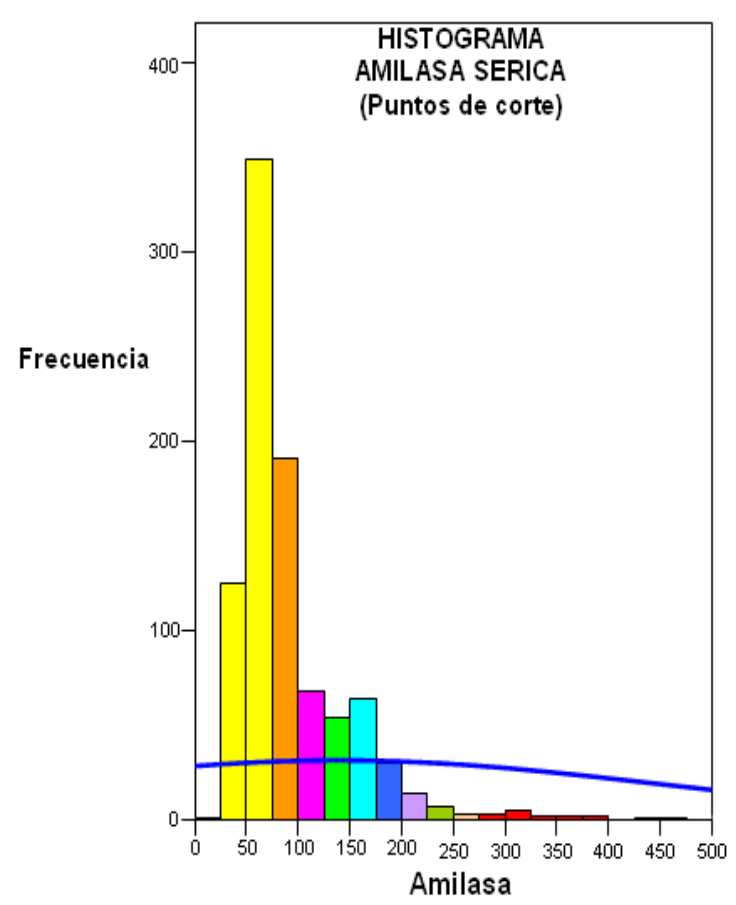

Tabla 1

Número de Pacientes por Categoría Diagnóstica Inicial

\begin{tabular}{|l|c|c|}
\hline Patología & Frecuencia & $\begin{array}{c}\text { Porcentaje } \\
\text { \% }\end{array}$ \\
\hline $\begin{array}{l}\text { Enfermedad } \\
\text { Acido péptica }\end{array}$ & 290 & 30.4 \\
\hline $\begin{array}{l}\text { Dolor Abdominal } \\
\text { en estudio }\end{array}$ & 281 & 29.4 \\
\hline $\begin{array}{l}\text { Enfermedad Diarreica } \\
\text { Aguda }\end{array}$ & 71 & 7.4 \\
\hline Pancreatitis & 67 & 7.0 \\
\hline $\begin{array}{l}\text { Abdomen Agudo } \\
\text { Inflamatorio }\end{array}$ & 66 & 6.9 \\
\hline Gastroenteritis & 52 & 5.4 \\
\hline Colelitiasis & 48 & 5.0 \\
\hline Colecistitis & 29 & 3.0 \\
\hline $\begin{array}{l}\text { Abdomen Agudo } \\
\text { Obstructivo }\end{array}$ & 17 & 1.8 \\
\hline Colon Irritable & 11 & 1.2 \\
\hline IVU & 10 & 1.0 \\
\hline Otras & 13 & 1.4 \\
\hline $\begin{array}{l}\text { Total } \\
\text { Fuente: Datos del Autor }\end{array}$ & 100 \\
\hline
\end{tabular}

Revista electrónica publicada por el Departamento de Farmacología de la Escuela de 


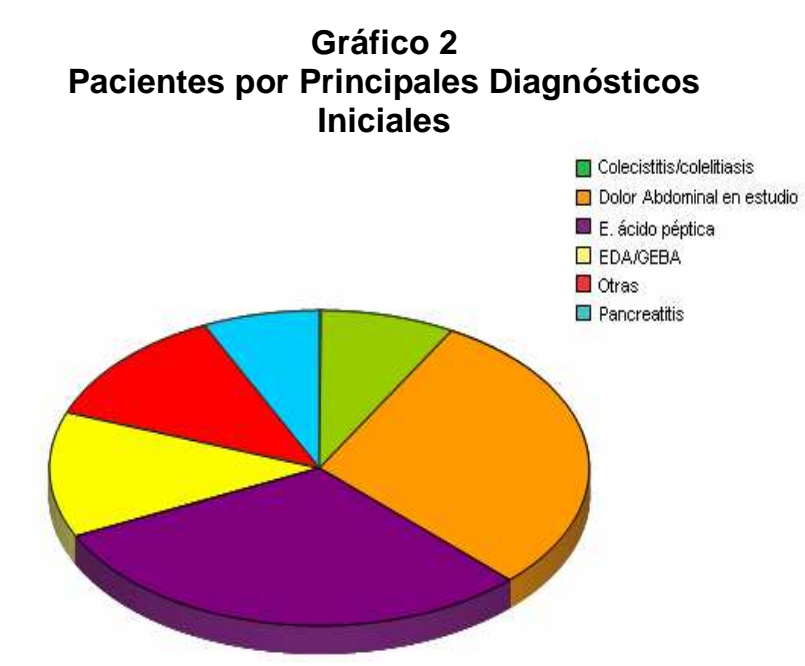

Fuente: Datos del Autor abdominal inespecífico y enfermedad diarreica aguda, otras como: gastroenteritis, colecistitis, apendicitis, colon irritable y pancreatitis se presentaron en menor proporción (Ver Tabla 3 y Gráfico 3).

En ciertas patologías la sospecha inicial varió considerablemente en relación a la definitiva, por ejemplo la pancreatitis aguda de inicio tuvo $7 \%$ de casos a diferencia del $5 \%$ que egresaron con este diagnóstico.

El $13.5 \%$ de los pacientes al egresar de Emergencia no tuvieron un diagnóstico certero, por lo que fueron catalogados como un cuadro de dolor abdominal inespecífico al momento del alta.

Tabla 2

Número de Pacientes por Categoría Diagnóstica Final

\begin{tabular}{|l|c|c|}
\hline Patología & Frecuencia & $\begin{array}{c}\text { Porcentaje } \\
\%\end{array}$ \\
\hline $\begin{array}{l}\text { Enfermedad } \\
\text { Acido péptica }\end{array}$ & 382 & 40 \\
\hline $\begin{array}{l}\text { Dolor Abdominal } \\
\text { Inespecífico }\end{array}$ & 129 & 13.5 \\
\hline $\begin{array}{l}\text { Enfermedad Diarreica } \\
\text { Aguda }\end{array}$ & 91 & 9.5 \\
\hline Pancreatitis & 53 & 5.5 \\
\hline $\begin{array}{l}\text { Apendicitis } \\
\text { Aguda }\end{array}$ & 51 & 5.3 \\
\hline Gastroenteritis & 69 & 7.2 \\
\hline Colelitiasis & 56 & 5.9 \\
\hline Colecistitis & 42 & 4.4 \\
\hline $\begin{array}{l}\text { Obstrucción } \\
\text { Intestinal }\end{array}$ & 8 & 0.8 \\
\hline Colon Irritable & 17 & 1.8 \\
\hline IVU & 18 & 1.9 \\
\hline Otras & 39 & 4.2 \\
\hline Total \\
Fuente: Datos del Autor
\end{tabular}

Fuente: Datos del Autor

$\begin{array}{llr}\text { Las principales patologías } & \text { que } \\ \text { presentaron los pacientes como } & \text { como } \\ \text { diagnóstico definitivo } & \text { fueron: } \\ \text { enfermedad ácido péptica, dolor }\end{array}$

\section{Gráfico 3}

Pacientes por Principales Diagnósticos Definitivos

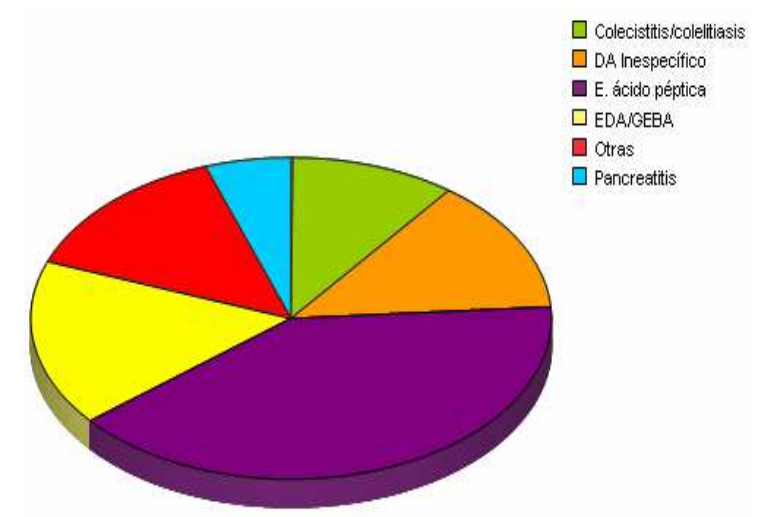

Fuente: Datos del Autor

En la Tabla 3 se muestran los resultados de validez y concordancia, presentándose los diez puntos de corte obtenidos con los valores de amilasa sérica y las medidas de probabilidad correspondientes a cada intervalo previamente establecido.

Revista electrónica publicada por el Departamento de Farmacología de la Escuela de 
Tabla 3

Validez Diagnóstica de la Amilasa Sérica

\begin{tabular}{|c|c|c|c|c|c|c|c|}
\hline & $\begin{array}{l}5 \\
9\end{array}$ & $\begin{array}{l}\mathbf{E} \\
\%\end{array}$ & $\begin{array}{r}\text { VP } \\
\%\end{array}$ & $\begin{array}{c}\text { VP- } \\
\%\end{array}$ & LR + & LR - & $\begin{array}{l}\text { Cohen } \\
\text { happa }\end{array}$ \\
\hline$=75$ & 100 & 52 & 10 & 100 & 2.11 & 278 & 0.10 \\
\hline $76-100$ & 100 & 73 & 18 & 100 & 381 & 390 & 023 \\
\hline 101-125 & 98 & 81 & 23 & 99 & 521 & 42.2 & 031 \\
\hline $126-150$ & 98 & 87 & 30 & 99 & 76 & 45.3 & 0.41 \\
\hline 151-175 & 96 & 94 & 48 & 99 & 16.3 & 24.4 & 0.51 \\
\hline $176-300$ & 96 & 97 & 68 & 99 & 37.7 & 253 & 0.78 \\
\hline $201-275$ & 94 & 98 & 83 & 99 & 85 & 17.1 & 0.87 \\
\hline $226-250$ & 92 & 99 & 92 & 99 & 2083 & 12.9 & 091 \\
\hline $251-275$ & 88 & 99 & 93 & 99 & 26627 & 86 & 090 \\
\hline$=275$ & 86 & 99 & 97 & 99 & 781.4 & 74 & 091 \\
\hline
\end{tabular}

Fuente: Datos del Autor

En el intervalo de amilasa sérica de 176 a $200 \mathrm{mg} / \mathrm{dl}$ (mejor punto de corte) se obtuvo una sensibilidad de $96 \%$ (IC 95\%: 86-99), especificidad de 97\% (IC 95\%: 96-98), valor predictivo positivo de $68 \%$ (IC 95\% 56-78), valor predictivo negativo de 99\% (IC 95\%: 99-100), LR+ de 37 , LR- de 25 y kappa de 0.78

El porcentaje de pacientes clasificados correctamente respecto a la prueba de oro en cada intervalo y el índice kappa (0.10 a 0.90) aumentaron al añadir un intervalo mayor de amilasa como punto de corte.

La probabilidad preprueba de padecer pancreatitis aguda es del 5 al $7 \%^{7}$, al obtener el índice de máxima verosimilitud positivo del valor de amilasa con el mejor punto de corte, se puede conseguir mediante un nomograma la probabilidad posprueba de tener dicha patología (Ver gráfico 4).

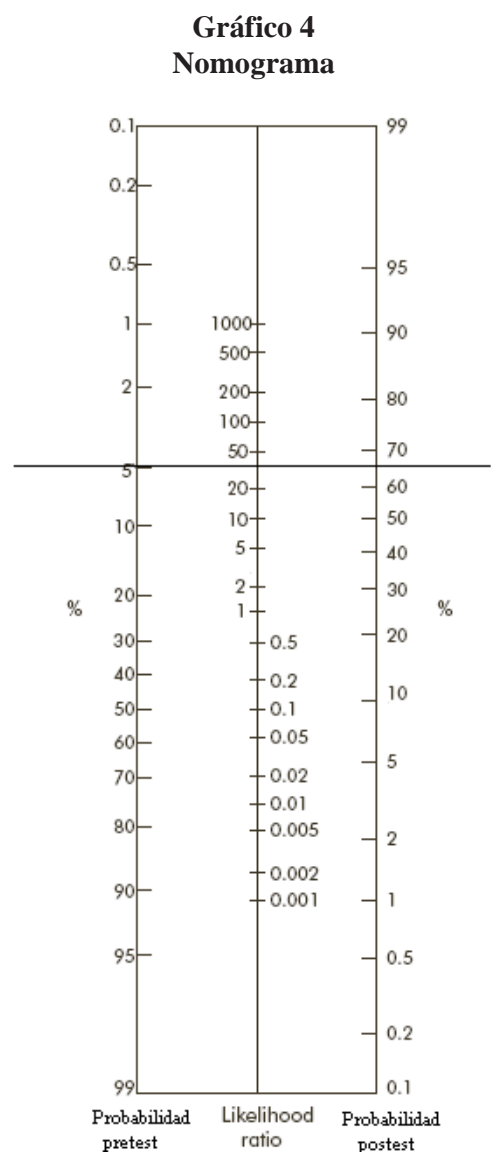

En la tabla 5 se muestra la frecuencia y proporción que presentaron los pacientes en relación a cada punto de corte, lo que permite establecer la distribución por intervalos en cada grupo de casos.

Revista electrónica publicada por el Departamento de Farmacología de la Escuela de Medicina de la Universidad de Costa Rica, 2060 San José, Costa Rica. @ All rights reserved. 
Tabla 4

Frecuencia de pacientes por Punto de Corte

\begin{tabular}{|c|c|c|}
\hline $\begin{array}{c}\text { Puntos de } \\
\text { corte } \\
\text { mg/dl }\end{array}$ & Frecuencia & Porcentaje \\
\hline$<\mathbf{7 5}$ & 490 & $51.4 \%$ \\
\hline $\mathbf{7 6 - 1 0 0}$ & 178 & $18.7 \%$ \\
\hline $\mathbf{1 0 1 - 1 2 5}$ & 67 & $7 \%$ \\
\hline $\mathbf{1 2 6 - 1 5 0}$ & 60 & $6.2 \%$ \\
\hline $\mathbf{1 5 1 - 1 7 5}$ & 60 & $6.2 \%$ \\
\hline $\mathbf{1 7 6 - 2 0 0}$ & 28 & $2.9 \%$ \\
\hline $\mathbf{2 0 1 - 2 2 5}$ & 13 & $1.4 \%$ \\
\hline $\mathbf{2 2 6 - 2 5 0}$ & 7 & $0.8 \%$ \\
\hline $\mathbf{2 5 1 - 2 7 5}$ & 3 & $0.3 \%$ \\
\hline$>275$ & 49 & $5.1 \%$ \\
\hline Total & 955 & $100 \%$ \\
\hline
\end{tabular}

Fuente: Datos del autor

En el gráfico 6 se muestra la curva ROC y el área bajo la curva que se obtuvo al establecer como punto de corte el valor de $176 \mathrm{mg} / \mathrm{dl}$ de amilasa sérica.

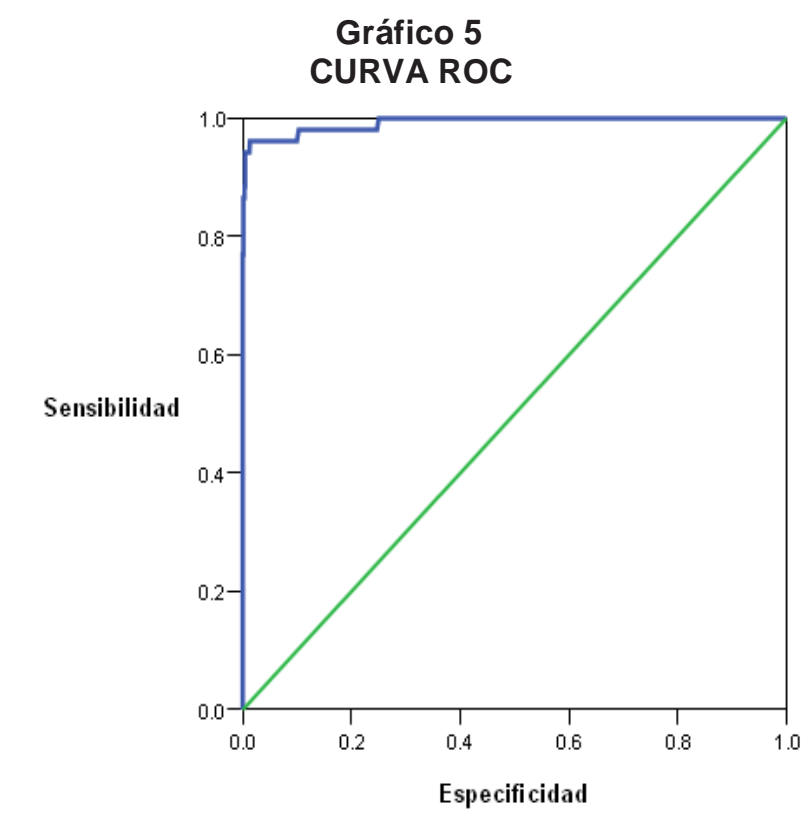

Fuente: Datos del Autor

Revista electrónica publicada por el Departamento de Farmacología de la Escuela de Medicina de la Universidad de Costa Rica, 2060 San José, Costa Rica. @ All rights reserved.
Area bajo la curva

(Amilasa $176 \mathrm{mg} / \mathrm{dl}$ )

\begin{tabular}{|c|c|c|}
\hline AUC & \multicolumn{2}{|c|}{ Intervalo de Confianza } \\
\hline 0.99 & 0.98 & 1.0 \\
\hline
\end{tabular}

\section{DISCUSIÓN}

El diagnóstico diferencial del dolor abdominal agudo (DAA), dentro del cual se encuentra la pancreatitis aguda (PA), plantea dificultades para el médico en el Servicio de Emergencia, a pesar del incremento del número de pruebas diagnósticas que se pueden utilizar[5].

Durante la investigación realizada a los pacientes con dolor abdominal y con sospecha de pancreatitis aguda, se pidió amilasa sérica como prueba de inicio junto a otros exámenes de laboratorio e imagen.

En el presente trabajo fueron investigadas las historias clínicas de 1012 pacientes (955 ingresaron al estudio), de los cuales el $5 \%$ terminaron con el diagnóstico definitivo de pancreatitis aguda, observándose casos de enfermedad ácido péptica, dolor abdominal inespecífico, gastroenteritis, entre otras, en las que la amilasa sérica no fue útil para su diagnóstico y manejo definitivo.

En este estudio la edad promedio de los pacientes fue 39 años (rango 16 a 65 años), cifra que es similar al valor de la mediana (38 años), lo que indica que no hubo distorsión de datos por los valores extremos, apreciándose un sesgo bajo (0.3) debido a la homogeneidad de la muestra en relación a esta variable, además no hubo mayor variación con 
respecto al promedio alcanzado (desviación estándar 13.8).

En lo que se refiere a la amilasa sérica, ésta presentó un promedio de $138 \mathrm{mg} / \mathrm{dl}$ (rango 24 a 5268), cifra que es distinta al valor de la mediana $(75 \mathrm{mg} / \mathrm{dl})$, lo que indica la gran distorsión que se observó en los datos, el sesgo (9.2) evidencia la heterogeneidad de la muestra en relación a la variable, por lo que al obtener un histograma se reflejó la asimetría hacia la derecha de los datos, existiendo una variación significativa con respecto a la media que se obtuvo (desviación estándar 307.2).

En un meta-análisis realizado por Butler y cols. (2002) sobre el uso de amilasa sérica en dolor abdominal agudo se obtuvo por arriba del rango establecido previamente una sensibilidad de $95 \%$ y especificidad de $89 \%$, en cambio con el mejor punto de corte presentó una sensibilidad de $95 \%$ y especificidad de $99 \%$ [16].

Se realizaron 10 puntos de corte para la validación diagnóstica de la prueba, en los que se estableció la variación que existe en relación a la amilasa obtenida y el diagnóstico final de pancreatitis aguda.

En este estudio, al aplicar la prueba con el valor estandarizado como positivo $(>100 \mathrm{mg} / \mathrm{dl})$, la amilasa sérica detectó adecuadamente a los pacientes con pancreatitis aguda (sensibilidad 98\%) pero con una especificidad de $81 \%$, teniendo una capacidad moderada de modificar la probabilidad posprueba de padecer pancreatitis aguda $(L R+5.21)$ y con una magnitud de concordancia débil (kappa 0.31) en relación a lo esperado.
La aplicación de la amilasa sérica en base del mejor punto de corte (176 $\mathrm{mg} / \mathrm{dl}$ ) permitió identificar a los pacientes con pancreatitis aguda con una sensibilidad y especificidad altas, con una magnitud de concordancia buena (kappa 0.78) y demostró que influirá de una manera decisiva en la probabilidad posprueba de padecer esta enfermedad $(\mathrm{LR}+37)$.

La curva ROC y el área bajo la curva (AUC 0.99) obtenidos, determinan que la amilasa sérica tiene una capacidad discriminativa alta para diagnosticar pancreatitis aguda.

En relación a estudios anteriores se obtuvo datos no concluyentes en la validación del uso de la amilasa sérica, ya que existen investigaciones que demuestran resultados contradictorios, concordantes o no en comparación a los resultados que se alcanzó $[1,14,18,19]$.

Es importante indicar que este estudio presenta limitaciones que deben ser consideradas. Por un lado, fue llevado a cabo en forma retrospectiva, cuando la manera ideal de validar una prueba diagnóstica es prospectiva. Sin embargo, la información requerida por el método estadístico utilizado era muy simple y se encontraba disponible en las historias clínicas en forma clara, aunque hubo un porcentaje mínimo de datos que no se pudo recoger por la falta de información o por la ausencia de historias clínicas en algunos casos.

De todas formas, la validación de la herramienta diagnóstica se desarrolló sobre un número adecuado de pacientes con el espectro de diagnósticos diferenciales que suele observarse habitualmente [7].

Revista electrónica publicada por el Departamento de Farmacología de la Escuela de 
Los objetivos previamente planteados en el protocolo de investigación fueron cumplidos, ya que se conoce la validez de esta prueba en un hospital del país, lo que permitirá el uso correcto y adecuado de la amilasa sérica frente a un paciente con dolor abdominal en el Servicio de Emergencia y que exista la posibilidad de que presente pancreatitis aguda.

A pesar de los resultados favorables obtenidos en la investigación, es importante indicar que el uso de la amilasa sérica debe ser complementario a una correcta valoración clínica de cada paciente, es útil como ayuda diagnóstica en los casos en que se tenga una sospecha inicial de pancreatitis aguda, para que la decisión definitiva permita el seguimiento y manejo adecuado en cada caso.

\section{CONCLUSIONES Y RECOMENDACIONES}

En pacientes que acuden a Emergencia con dolor abdominal agudo y sospecha de posible pancreatitis aguda es válida la medición de amilasemia como prueba inicial de ayuda diagnóstica.

La cifra promedio en nuestro país que se usa para determinar si la prueba de amilasa sérica es positiva es un valor mayor a $100 \mathrm{mg} / \mathrm{dl}$, punto de corte en que es sensible pero poco específica en la detección de pacientes con pancreatitis aguda.

Para alcanzar una mayor exactitud en el diagnóstico de pancreatitis aguda, se necesita un valor de amilasa mayor a $175 \mathrm{mg} / \mathrm{dl}$ (mejor punto de corte), que junto al análisis clínico y otros exámenes de laboratorio e imagen complementarios permitirán tener mayor certeza al valorar un paciente con dolor abdominal y cifras elevadas de amilasemia.

Se recomienda la medición de amilasa sérica como ayuda diagnóstica inicial en pacientes con dolor abdominal agudo, su uso debe estar asociado a una correcta valoración y sospecha clínica, ya que va a complementar la información obtenida de una historia clínica adecuada.

Es necesario establecer parámetros y puntos de corte válidos para la interpretación correcta del valor de amilasemia en pacientes con sospecha de pancreatiis aguda.

Sería útil realizar otros estudios en el país con pruebas de ayuda diagnóstica de uso común, ya que así se podrá crear protocolos de manejo adaptados a su realidad, que permitan un mejor uso de los recursos disponibles, lo que ayudará a mejorar el cuidado del paciente y disminuir costos con una atención de calidad.

Es importante que durante la realización de un estudio de validación diagnóstica se utilice métodos estadísticos adecuados que posibiliten obtener datos de calidad, así se podrá aplicar en la práctica diaria pruebas exámenes de laboratorio en los que se conoce sus índices de probabilidad, verosimilitud y concordancia, que permitan luego de su análisis una mayor capacidad de decisión por parte del médico, para tener un sustento claro y conciso de lo que se va a realizar en favor de cada paciente.

Revista electrónica publicada por el Departamento de Farmacología de la Escuela de 


\section{Referencias}

1. Dekay ML, Asch DA. Is the Defensive Use of Diagnostic Tests Good for Patients, or Bad? Med Decis Making 1998;18:19-28.

2. Verstappen W, Smeele I, Hermsen J. Effect of a Practice-Based Strategy on Test Ordering Performance of Primary Care Physicians: A Randomized Trial. JAMA 2003;289:2407-12.

3. Goodman PE. Acute Abdominal Pain. En: Cline DM, Ma J, eds. Emergency Medicine $5^{\text {th }}$ ed. NC: McGraw-Hill;2001. Libro electronic.

4. Zeller JL, Burke AE. Acute Abdominal Pain. JAMA 2006;296:1800 (Letter).

5. Mulholland M, Sweeney J. Approach to the patient with acute abdomen. En: Yamada T, Alpers DH, Laine L, Powel D, eds. Yamada's Textbook of Gastroenterology 4th ed. PA: Lippincott Williams;2003. Libro electronic.

6. Silen William. Dolor Abdominal. En: Braunwald E, Fauci A, Kasper. eds. Principios de Medicina Interna de Harrison 15 $15^{\mathrm{a}}$ ed. DF: McgrawHill;2002:83-85.

7. Clavè P, Blanco I, Nabau N. Amylase, Lipase, Pancreatic Isoamylase, and Phospholipase A in Diagnosis of Acute Pancreatitis. Clin Chem 1995;41:112934.

8. Kearney DJ. Approach to the Patient with Gastrointestinal Disorders. En: Friedman SL, McQuaid KR, eds. Current Diagnosis and Treatment in Gastroenterology 2nd ed. NY: McGrawHill;2002. Libro electronic.

9. Fischer J, Nussbaum MS. Manifestations of Gastrointestinal Disease. En: Seymour I, Schwartz ed. Principles of
Surgery $7^{\text {th }}$ ed. NY: McGraw-Hill;1999. Libro electronic.

10. Britton J. The acute abdomen. En: Morris P, William C, eds. Oxford Textbook of Surgery. 2nd ed. Turín: Oxford University Press;2001. Libro electrónico.

11. Hedstrom J. Pancreatitis may occur with a normal amylase concentration in hypertriglyceridaemia. 1996;313:1265 (Letter).

12. Topazian M, Gorelick FS. Acute pancreatitis. En: Yamada T, Alpers DH, Laine L, Powel D. Yamada's Textbook of Gastroenterology $4^{\text {th }}$ ed. PA: Lippincott Williams; 2003. Libro electrónico .

13. Carroll JK. Acute Pancreatitis: Diagnosis, Prognosis, and Treatment. AAFP 2007; 75:1513-20.

14. Muñoz A. Diagnosis and Management of Acute Pancreatitis. AAFP 2000;62:164174.

15. Timothy J, Uzbielo Alison. Point-of-care Urine Trypsinogen Testing for the Diagnosis of Pancreatitis. Acad Emerg Med. 2007;14:29-34.

16. Butler J, Mackway-Jones K. Serum amylase or lipase to diagnose pancreatitis in patients presenting with abdominal pain. Emerg Med J. 2002;19:430-31.

17. Grendell JH. Acute Paancreatitis. En: Friedman SL., McQuaid KR, eds. Current Diagnosis and Treatment in Gastroenterology $2^{\text {nd }}$ ed. NY: McGrawHill; 2002. Libro electronic.

18. Beckingham IJ., Bornman PC. ABC of diseases of liver, pancreas, and biliary system: Acute pancreatitis. BMJ 2001;322:595-98.

Revista electrónica publicada por el Departamento de Farmacología de la Escuela de 
19. Kingsnorth A, O’Reilly D. Acute pancreatitis. BMJ 2006;332:1072-76.

20. Frank B, Gottlieb K. Amylase normal, lipase elevated: is it pancreatitis? A case series and review of the literature. Am J Gastroenterol 1999;94:463-469.

21. Kemppainen EK, Hedstrum JI. Rapid measurement of urinary trypsinogen-2 as a screening test for acute pancreatitis. N Engl J Med 1997;336:1788-93.

22. Lyon C, Clark DC. Diagnosis of Acute Abdominal Pain in Older Patients. AAFP 2006;74:1537-44.

23. Shomron B, Farfel Z. GastroenteritisAssociated Hyperamylasemia: Prevalence and Clinical Significance. Arch Inter Med 2002;162:689-92.

24. Dawson B, Trapp R. Bioestadística Médica. Manual Moderno. $2^{\mathrm{a}}$ ed. México DF. Manual Moderno SA;1999:275-294.

25. Raje ev S, Callum F. Hyperamylasaemia: not the usual suspects. BMJ 2005; 331:890-891.

26. Frederick $M$, Joseph $A$. Biostatics in Clinical Medicine. $3^{\text {rd }}$ ed. PA: Mc GrawHill; 1994.

27. Knapp R, Miller C. Clinical Epidemiologics and Biostatistics. 1a ed. William and Wilkins eds. PA: Harwal Publishing CO;1992.

28. Thomas A, Secic M. How To Report Statistics in Medicine. Evidence Based Medicine 2007;12:90-91.

29. Altman D.G., Bland J.M. Statistics Notes: Diagnostic tests 1: sensitivity and specificity. BMJ 1994;308:1552.

30. Fajardo GA, Yamamoto KL. Utilidad de las curvas de sensibilidad $y$ especificidad conjunta en la aplicación de una prueba de diagnóstico. Salud Pública Mex 1994;36:311-317.
31. Cerda J, Villaroel L. Evaluación de la concordancia inter-observador en investigación pediátrica: Coeficiente de Kappa. Rev Chil Pediatr 2008;79:54-58.

32. Escobar Koziel LF. Investigación Científica para Médicos. $3^{\mathrm{a}}$ ed. Quito: Cuadernos Académicos Facultad de Medicina PUCE; 1999:157-177.

33. Burgueño MJ, García-Bastos JL, González-Buitrago JM. Las curvas ROC en la evaluación de las pruebas diagnósticas. Med Clin (Barc) 1995;104:661-670.

34. Metz CE, Herman BA, Shen, J. Maximum likelihood estimation of receiver operating characteristic (ROC) curves from continuously distributed data. Statist Med 1998;17:1033-1053.

35. Forsmark CE, Baillie J. AGA Institute Technical Review on acute pancreatitis. Gastroenterology 2007;132:2022-2044.

36. Raje ev S, Callum F. Hyperamylasaemia: not the usual suspects. BMJ 2005; 331:890-891.

37. Williams L. Chemistry Studies. En: Zang, Sheryl $M$, and Allender, Judith $A$ eds. Manual of Laboratory and Diagnostic Tests 6th Edition. PA: Lippincott \& Raven; 2000. Libro electronic.

38. Altman D.G., Bland J.M. Statistics Notes: Diagnostic tests 2: predictive values. BMJ 1994;309:102.

39. Mackinnon, A. (2000) A spreadsheet for the calculation of comprehensive statistics for the assessment of diagnostic tests and inter-rater agreement. Computers in Biology and Medicine. 30(3):127-134.

\section{Correspondencia:}

Diego Viteri Cevallos

Correo electrónico:diegoviteri83@hotmail.com

Revista electrónica publicada por el Departamento de Farmacología de la Escuela de 